\title{
Pleural Tuberculosis and its Treatment Outcomes
}

\author{
Amer Hayat Khan ${ }^{1^{*}}$, Syed Azhar Syed Sulaiman ${ }^{1}$, Abdul Razak Muttalif ${ }^{2}$, \\ Mohamed Azmi Hassali ${ }^{3}$, Hadeer Akram ${ }^{1}$, Syed Wasif Gillani and Syed Wasif \\ Gillani ${ }^{1}$
}

${ }^{1}$ Department of Clinical Pharmacy, School of Pharmaceutical Sciences, University Sains Malaysia, 11800 Penang, ${ }^{2}$ Department of Respiratory Medicine, Penang Hospital, Penang, ${ }^{3}$ Discipline of Social \& Administrative Pharmacy, School of Pharmaceutical Sciences, University Sains Malaysia, 11800 Penang, Malaysia, ${ }^{4}$ Discipline of Pharmacy, Monash University, Bandar Sunway, Selangor, Malaysia

*For correspondence: Email: amerhayat@ymail.com; Tel: 006-017-3860275; Fax: 006046570017

Received: 19 July 2012

Revised accepted: 23 June 2013

\begin{abstract}
Purpose: To evaluate the incidence, treatment and clinical outcomes of tuberculosis pleuritis at a hospital in the state of Penang, Malaysia.

Methods: A retrospective study was conducted in Hospital of Penang, Malaysia. Patient records were reviewed retrospectively to identify patients with confirmed diagnosis of tuberculous pleuritis from January 2006 to December 2008. Chest x-ray (CXR) and pleural biopsy were carried out on all patients. Directly observed therapy (DOT) was given to all patients. Data were analyzed using SPSS version 16. Results: Of 1548 tuberculosis cases, 80 (5.2\%) patients had tuberculous pleuritis. The mean age of the patients was $35.4 \pm 12.87$ years, with a male to female ratio of $3.4: 1$. Ethnically, a plurality $(n=30$, $37.7 \%$ ) of cases among tuberculosis pleuritis patients were Chinese, followed by Malay (31.2\%). Out of the 80 patients with tuberculous pleuritis, $10(12.5 \%)$ also had diabetes mellitus, and $8(10.0 \%)$ HIVIAIDS. Fever, cough, chest pain and shortness of breathing were the most frequently reported symptoms. Treatment success rate was 1.558 times higher among TB group than pleuritis TB group (Odds ratio, $95 \% \mathrm{Cl}, 1.06-2.59, p=0.025$ ).

Conclusion: The incidence of TB pleuritis was gender- and race-related, with DM and HIV the most commonly reported risk factors. Treatment success rate was higher among pulmonary TB group than in those with TB pleuritis (extra pulmonary TB).
\end{abstract}

Keywords: Tuberculosis, Pleuritis, HIVIAIDS, Biopsy, Pulmonary.

Tropical Journal of Pharmaceutical Research is indexed by Science Citation Index (SciSearch), Scopus, International Pharmaceutical Abstract, Chemical Abstracts, Embase, Index Copernicus, EBSCO, African Index Medicus, JournalSeek, Journal Citation Reports/Science Edition, Directory of Open Access Journals (DOAJ), African Journal Online, Bioline International, Open-J-Gate and Pharmacy Abstracts

\section{INTRODUCTION}

Tuberculosis (TB) is a leading source of morbidity and mortality throughout the world. It is estimated that in 2011, there was a total of 8.7 million new cases and 1.4 million deaths from TB [1]. Although the African region has the highest estimated incidence (356 per 100,000 populations per year), the majority of patients with TB live in the most populous countries of the Asian subcontinent, which accounts for nearly half of the new cases that arise yearly [2]. The frequency of pleural involvement in TB has been variously reported to range from $4 \%$ in United States to $23 \%$ in Spain) $[3,4]$.

TB pleural effusion is the second most common form of extrapulmonary tuberculosis (EPTB), only less frequent than lymph node TB. TB pleural effusion is increasingly recognized, even in developed nations [5], as the incidence of EPTB has more than doubled following HIV pandemic. The incidence of TB pleural effusions in human immunodeficiency virus / acquired immuno- 
deficiency syndrome (HIVIAIDS) has been variously reported to range from 15 to $90 \%$ with effusion being more common in patients with higher CD4+ counts [6-8].

In some parts of the world, tuberculosis remains the most frequent cause of pleural effusion in the absence of an obvious pulmonary lesion. Diagnosis of tuberculosis in pleural effusion can be made by pleural fluid culture. Pleural biopsy can also be used to diagnose TB. When microscopic examination of biopsy specimen is combined with culture, diagnosis can be made in $90 \%$ of patients, especially with the use of multiple biopsies [9].

Malaysia, a multicultural and multiethnic country, has a population slightly above 26 million and an area of $33,000 \mathrm{~km}^{2}$. Tuberculosis is among the top five communicable diseases in the country with an incidence rate of 62.56 per 100,000 and mortality rate of 5.37 per 100,000 [10]. The State of Penang is the eighth most populous state in Malaysia and among the top five states with the highest tuberculosis burden [10]. Pulmonary tuberculosis has been the disease of interest for researchers but little work has been done on extra-pulmonary tuberculosis, especially, tuberculous pleura. The current study was designed to expand the current knowledge by gathering baseline data on the incidence, diagnosis, complications, management and treatment outcomes of tuberculous pleural effusion in a teaching hospital.

\section{Methods}

A retrospective study was conducted among patients visiting the respiratory clinic, Penang Hospital. Penang is one of thirteen states and is geographically situated in northern Malaysia. It is a multicultural state comprising of Malay (42.5 $\%)$, Chinese $(46.5 \%)$, Indian $(10.6 \%)$ and minorities $(0.4 \%)$, with an estimated population of 1.5 million [11].

A total of 1548 cases were registered for tuberculosis treatment from $1^{\text {st }}$ January 2006 to December 2008 at the respiratory diseases clinic of Penang Hospital. This clinic is a tertiary level referral centre for respiratory diseases in the state of Penang, Malaysia. Any person with a respiratory problem in the state of Penang can attend this centre with a physician's referral.

A retrospective evaluation of the medical records of patients with confirmed diagnosis of tuberculosis pleura was carried out. From each medical case file, the patient's history, physical parameters, chest radiographs and laboratory investigation was reviewed in order to obtain maximum information about the type and severity of TB. In addition, demographic factors, life style (smoking habit and alcohol use) and clinical characteristics were recorded. Among clinical characteristics, co-morbid medical complications (such as diabetes mellitus, HIV, asthma and chronic obstructive pulmonary disease COPD), medications for therapy and therapeutic outcomes were recorded, based on Chi-square and Odds ratio. The study protocol was approved by Clinical Research Center (CRC), Penang Hospital and Ministry of Health, Malaysia (ref no. KKM/NIHSEC/08/0804P87-177).

\section{Statistical analysis}

Data with quantitative variables were expressed by mean \pm standard deviation (SD) and range while qualitative variables were estimated by frequency and percentage. Chi square $\left(X^{2}\right)$ and Odds ratio were applied to compare outcomes, and differences were considered significant at $p$ $<0.05$. The data were analyzed using SPSS ${ }^{\circledR}$ software, version 16 .

\section{RESULTS}

During the period, there was a high prevalence of TB pleuritis (EPTB) among all TB cases, with lymph node involvement $(n=109,7.1 \%)$ [12] being the commonest, followed by pleurisy ( $\mathrm{n}=$ $80(5.2 \%)]$. Table 1 shows the demographic features of pleural tuberculosis; it was more prevalent among younger age groups, $(n=60$, $75.0 \%$ ), i.e., < 46 years. The mean age of the study subjects was $35.4 \pm 12.9$ (range $5-71$ years) and male to female ratio was $3.4: 1$. Three $(3.8 \%)$ of the patients with pleural TB were referred from Penang prison. Other comorbidities encountered include diabetes mellitus $(n=10,12.5 \%)$, HIVIAIDS $(n=8,10 \%)$ and asthma ( $\mathrm{n}=2,2.5 \%$ ). Other demographic parameters are shown in Table 1.

A majority, $73(91.2 \%)$, of patients reported cough, 69 (86.2 \%) fever, 51 (63.8\%) chest pain and $65 \%$ had night sweats. Details of the symptoms reported are listed in Table 2.

Table 3 shows chest $x$-ray and laboratory results. The disease was mostly a primary infection rather than a reactivation of co-existing pulmonary tuberculosis (past history and/or family history of the disease was absent in $93.8 \%$ of cases, and evidence of parenchyma lung lesions were absent in $78 \%$ of cases). Right pleural effusion was slightly more common than left pleural effusion (48.6\% compared to 27.5 $\%)$. Up to $3.8 \%$ of cases had bilateral pleural 
effusion. ESR was elevated in a majority of cases (> $40 \mathrm{~mm} / \mathrm{h}$ in $56.2 \%$ ). Pleural biopsies for culture and sensitivity were positive in 49 (61.3 $\%)$ of the cases.

Table 1: Demographic profile of patients $(N=80)$

\begin{tabular}{|c|c|}
\hline Parameter & $\mathbf{N}(\%)$ \\
\hline $\begin{array}{l}\text { Sex ratio (M: F) } 3.44: 1 \\
\text { Range of age ( } 5-71 \text { years) } \\
\text { Mean age } 35.4 \pm 12.87\end{array}$ & $\mathrm{~N}=80 n(\%)$ \\
\hline \multicolumn{2}{|l|}{ Gender } \\
\hline Male & $62(77.5)$ \\
\hline Female & $18(22.5)$ \\
\hline \multicolumn{2}{|l|}{ Race } \\
\hline Malay & $25(31.2)$ \\
\hline Chinese & $30(37.7)$ \\
\hline Indian & $19(23.7)$ \\
\hline Indonesian & $2(2.5)$ \\
\hline Others & $4(4.8)$ \\
\hline \multicolumn{2}{|l|}{ Age group } \\
\hline$<35$ years & $36(45.0)$ \\
\hline $35-45$ years & $24(30.0)$ \\
\hline$>45$ years & $20(25.0)$ \\
\hline \multicolumn{2}{|l|}{ Contact history } \\
\hline Yes & $3(3.8)$ \\
\hline No & 75 (93.8) \\
\hline Unknown & $2(2.5)$ \\
\hline \multicolumn{2}{|l|}{ Place } \\
\hline Hospital & $77(96.2)$ \\
\hline Prison & $3(3.8)$ \\
\hline \multicolumn{2}{|l|}{ Geographical location } \\
\hline Urban Area & $49(61.2)$ \\
\hline Rural Area & $31(38.8)$ \\
\hline \multicolumn{2}{|l|}{ Diabetes mellitus } \\
\hline Yes & $10(12.5)$ \\
\hline No & $70(87.5)$ \\
\hline \multicolumn{2}{|l|}{ HIV } \\
\hline Yes & $8(10.0)$ \\
\hline No & $72(90.0)$ \\
\hline \multicolumn{2}{|l|}{ Asthma } \\
\hline Yes & $2(2.5)$ \\
\hline No & $78(97.5)$ \\
\hline \multicolumn{2}{|l|}{ COPD* } \\
\hline Yes & $3(3.7)$ \\
\hline No & $(96.3)$ \\
\hline \multicolumn{2}{|l|}{ Drinks alcohol } \\
\hline Yes & $16(20.0)$ \\
\hline No & $28(35.2)$ \\
\hline Unknown & $29(36.2)$ \\
\hline Ex-Drinker & $7(8.8)$ \\
\hline \multicolumn{2}{|l|}{ Smokes } \\
\hline Yes & $27(33.8)$ \\
\hline No & $35(48.8)$ \\
\hline Unknown & $11(13.8)$ \\
\hline Ex-Smoker & $7(8.8)$ \\
\hline
\end{tabular}

${ }^{*}$ Chronic obstructive pulmonary disease

\section{Management and outcomes}

All patients received directly observed therapy (DOTS) from six to nine months. Two months ethambutol, isoniazid, rifampicin and pyrazinamide with four months biweekly isoniazid and rifampicin (2EHRZ+4H2R2) were the therapeutic combination used for the majority 27 $(33.8 \%)$ of the patients. Based on the clinical outcomes, the duration of intensive phase was increased to three months in $33.2 \%$ patients while the treatment success rate for TB group being higher than pleuritis TB group (odd ratio = 1.558, 95\% Cl, $1.06-2.59, p=0.025)$. Six (7.5 $\%$ patients defaulted the treatment and seven $(8.7 \%)$ patients were recorded as deceased during the course of therapy, as shown in Table 4.

Table 2: Symptoms reported by patients with tuberculous pleuritis $(\mathrm{N}=80)$

\begin{tabular}{lc}
\hline Sign/symptom & $\mathbf{N}(\%)$ \\
\hline Fever & $73(91.2)$ \\
Cough & $69(86.2)$ \\
Sputum & $37(46.2)$ \\
Hemoptysis & $33(41.2)$ \\
Weight loss & $45(56.2)$ \\
Loss of appetite & $42(52.5)$ \\
Shortness of breath & $49(61.2)$ \\
Chest pain & $51(63.8)$ \\
Night sweating & $52(65.0)$ \\
Others & $4(4.8)$ \\
\hline
\end{tabular}

Table 3: Laboratory test results for patients $(N=80)$

\begin{tabular}{ll}
\hline Test parameter & N (\%) \\
\hline Chest $x$-ray & $39(48.6)$ \\
Right effusion & $22(27.5)$ \\
Left effusion & $3(3.8)$ \\
Bilateral pleural effusion & $8(10.0)$ \\
Consolidation & $5(6.3)$ \\
Fibrosis & $3(3.8)$ \\
Cavitation & $7(8.8)$ \\
Sputum for AFB (direct smear) & $73(91.2)$ \\
Positive & \\
Negative & $25(31.3)$ \\
Positive & $55(68.7)$ \\
Negative & $14(17.6)$ \\
Yes & $66(82.4)$ \\
No & $49(61.3)$ \\
Pleural biopsy of AFB direct smear & $31(38.7)$ \\
Pleural biopsy of AFB culture sensitivity & \\
No & $9(11.3)$ \\
ESR ${ }^{* \star}$ (mm/h) & $26(32.5)$ \\
$<20$ & $45(56.2)$ \\
$20-40$ &
\end{tabular}

\section{DISCUSSION}

There has been a rise in the prevalence of TB throughout the world with a propensity for extrapulmonary involvement. Similarly, HIVIAIDS cases continue to grow in numbers.

in older age groups, suggesting that it may be

Trop J Pharm Res, August 2013;12 (4): 
Table 4: Categorization of patients according to treatment outcomes

\begin{tabular}{|c|c|c|c|c|c|}
\hline $\begin{array}{l}\text { Overall treatment } \\
\text { outcomes }\end{array}$ & $\begin{array}{l}\text { No. of TB patients } \\
\text { without pleuritis }\end{array}$ & $\begin{array}{l}\text { No. of TB } \\
\text { patients with } \\
\text { pleuritis }\end{array}$ & $P$-value & Odd Ratio & $95 \% \mathrm{Cl}$ \\
\hline Successfully treated & $1019(69.5 \%)$ & $48(59.9 \%)$ & 0.025 & 1.558 & $1.061-2.590$ \\
\hline Patient defaulted & $81(5.5 \%)$ & $6(7.4 \%)$ & 0.533 & -- & -- \\
\hline Transferred out & $77(5.2 \%)$ & $5(6.2 \%)$ & 0.122 & -- & -- \\
\hline Patient expired & $120(8.2 \%)$ & $5(6.2 \%)$ & 0.668 & -- & -- \\
\hline Treatment continued & $171(11.5 \%)$ & $16(20.1 \%)$ & 0.081 & -- & -- \\
\hline
\end{tabular}

${ }^{*} A$ patient who has been transferred to another recording and reporting unit and for whom the treatment outcome is not known;

${ }^{* *} A$ patient who dies for any reason during the course of treatment

Published data from some developed countries indicate that pleural tuberculosis is more frequent due to reactivation of the disease $[3,7,8,13]$. However the situation in developing countries is not clear. In the current study, we found that the disease is mainly a primary one rather than a reactivation of previous parenchymal lung tuberculosis. In addition, we found that pleural tuberculosis tends to affect younger age groups ( $<45$ years) and this more pronounded in males. These findings may be explained partly by the reduction in incidence of tuberculosis in developed world [5]. The unilateral tuberculous effusion reported in the present study confirms previous findings of right-sided predominance $[3,14]$.

One recent study from Spain [15] that described pleural tuberculosis in children showed that history of contact with a tuberculous case was positive in $25.7 \%$ of patients, but in the present study, we found only $3.8 \%$ of our patients had such history, indicating that contact with tuberculous cases seems to be less evident in adults.

Pleural tuberculosis is still a difficult disease to diagnose; pleural biopsy remains the gold standard for the diagnosis of pleural tuberculosis $[5,6]$. In a study of 42 patients with tuberculous effusions, closed needle biopsy achieved a diagnostic sensitivity of $79 \%$ when histology and culture was declared a reliable tool for diagnosis [16]. Seibert et al reported a yield of $66.7 \%$ from closed pleural biopsy cultures and $84.6 \%$ from histology [7]. Pleural tissue simultaneously sent for mycobacterial culture in addition to histology may increase diagnostic yield further. The diagnostic yield has been reported to rise to $90 \%$ when closed pleural biopsies are cultured [17].

The diagnosis of tuberculous pleural effusion is an indication for treatment with anti-tuberculous chemotherapy. Standard short course chemotherapy with isoniazid, rifampicin, pyrazinamide and ethambutol followed by 4 months of isoniazid and rifampicin is sufficient in the majority of cases [18]. Following the initiation of the standard 4-drug regimen mentioned above, subsequent treatment should be guided according to the results of drug sensitivity testing when they become available to the clinician. The incidence of residual pleural thickening and functional squeal can be reduced by effective anti-tubercular chemotherapy [19]. Although studies by Dutt et al supported the effectiveness of a 6-month regimen of isoniazid and rifampicin in both pulmonary and pleural disease, this is generally not advised due to the problems associated with drug resistance [8]. Paradoxical enlargement of the effusion has been reported after the commencement of anti-tuberculous chemotherapy similar to that seen in intra-cranial tuberculomas and this can require drainage, in some cases, for symptom relief [20].

A number of studies suggest increased incidence of pleural involvement in AIDS cases diagnosed with active tuberculosis. In a study comparing 963 HIV positive adults with pulmonary TB with 1000 HIV negative age-matched controls, there was a significantly higher incidence of pleural effusions (16 vs. $6.8 \%$ ) [21]. In the western world, a study in South Carolina, USA appeared to show increased rates of pleural TB in AIDS cases (11\%) compared to non-AIDS cases (6\%) [22].

There is also evidence to suggest that HIV coinfection alters the clinical presentation of TB pleurisy. The duration of the presenting illness tends to be longer in a substantial number of such cases compared with that seen in HIV negative individuals. A study from Tanzania found that HIV positive patients tend to present with more constitutional symptoms such as dyspnoea, fevers, night sweats, Treatment of tuberculous pleurisy in HIV positive co-infection is generally successful with a good outcome in most cases. In a series of 22 patients, there were 2 deaths reported and only 1 after antituberculous chemotherapy was commenced [21]. The current study's main focus is on pleurisy TB (EPTB) but comparison was made with PTB, to get a clear picture based on treatment outcomes. It was found that treatment completion rate was 
$48(60.0 \%)$, default and failure rate were 6 $(7.4 \%)$ and $5(6.2 \%)$ respectively. Deaths were found in $5(6.2 \%)$ cases; among these 5 cases, 4 were affected with co-morbid condition (3 patients were pleuritis TB plys HIVIAIDS and 1 with diabetes mellitus). Similar default and failure rate have been reported by Sharma et al [23].

\section{Limitations of the study}

All registered cases from January 2006 to December 2008 in the cited clinic were part of study. Due to the retrospective nature of the study, some social factors were, however, considered "Unknown" where they were unconfirmed.

\section{CONCLUSION}

The incidence of pleura TB was higher in males than in females and higher in Chinese race than other ethnic group. Fever, cough, chest pain, shortness of breathing, night sweat were clinically proven symptoms. The study confirms early detection by simple tests and ensuring complete treatment using direct observe therapy short course (DOTS) strategy. DOTS strategy is an effective treatment modality for TB pleurisy, achieving a high treatment completion rate, low default and failure rates. HIVIAIDS, diabetes mellitus, COPD and asthma are co-morbidity which makes treatment more complicated.

\section{ACKNOWLEDGMENT}

One of the authors (Amer Hayat Khan) would like to acknowledge the financial support from the Institute of Postgraduate Studies, Universiti Sains Malaysia to carry out this Study.

\section{REFERENCES}

1. Sudre P, Ten Dam G, Kochi A. Tuberculosis: a global overview of the situation today. Bull WHO. 1992; 70(2):149-159.

2. Aktogu S, Yorgancioglu A, Cirak K, Kose T, Dereli SM. Clinical spectrum of pulmonary and pleural tuberculosis: a report of 5,480 cases. Eur RespJ. 1996; 9(10): 2031-2035.

3. Moudgil H, Sridhar G, Leitch AG. Reactivation disease: the commonest form of tuberculous pleural effusion in Edinburgh, 1980-1991. Resp Med. 1994; 88(4): 301-304.

4. Antoniskis D, Amin K, Barnes PF. Pleuritis as a manifestation of reactivation tuberculosis. $A m \mathrm{~J}$ Mede. 1990; 89(4): 447-450.
5. Morehead RS. Tuberculosis of the pleura. Southern Med J. 1998; 91(7): 630.

6. Chan CHS, Arnold M, Chan CY, Mak TWL, Hoheisel GB. Clinical and pathological features of tuberculous pleural effusion and its long-term consequences. Respiration. 1991; 58(3-4): 171175.

7. Seibert AF, Haynes $J r J$, Middleton R, Bass $J r J B$. Tuberculous pleural effusion. Twenty-year experience. Chest. 1991;99(4):883-886.

8. Dutt AK, Moers D, Stead WW. Tuberculous pleural effusion: 6-month therapy with isoniazid and rifampin. Am. J. Respir. Crit. Care Med.. 1992; 145(6): 1429-1432

9. Friedman LN. Tuberculosis: current concepts and treatment. Informa HealthCare; 2001; p 518.

10. Malaysia MoH. Health Facts 2006; July 2007. [cited 2012 February 23]. Available from http://www.moh.gov.my/images/gallery/stats/heal_f act/health_facts_2006.pdf.

11. Institute S-EER. Penang Statistics. Quarter 2, 2007. [cited 2012 February 23]. Available from http://www2.seri.com.my/Penang\%20Statistics/200 7/Q2-April-June-2007 1.pdf.

12. Khan $A H$, Sulaiman $S A$, Muttalif AR, Hassali MA, Khan TM. Tuberculous Lymphadenitis at Penang General Hospital, Malaysia. Med Princ Pract. 20(1): 80-84.

13. Epstein DM, Kline LR, Albelda SM, Miller WT. Tuberculous pleural effusions. Chest. 1987; 91(1): 106-109.

14. Sibley JC. A study of 200 cases of tuberculous pleurisy with effusion. Am Rev Tuberc 1950; 62(3): 314-324

15. Merino JM, Carpintero I, Alvarez T, Rodrigo J, SÃ $j$ nchez J, Coello JM. Tuberculous Pleural Effusion in Children*. Chest. 1999; 115(1): 26-30.

16. Diacon $A H$, Van de Wal BW, Wyser C, Smedema JP, Bezuidenhout J, Bolliger CT, et al. Diagnostic tools in tuberculous pleurisy: a direct comparative study. Eur Resp J. 2003; 22(4): 589-591.

17. Levine $H$, Metzger W, Lacera D, Kay L. Diagnosis of tuberculous pleurisy by culture of pleural biopsy specimen. Arch Inter Med. 1970; 126(2): 269-271

18. Joint T. Chemotherapy and management of tuberculosis in the United Kingdom: recommendations 1998. Thorax. 1998; 53(7): 536-548

19. Lee $\mathrm{CH}$, Wang WJ, Lan RS, Tsai YH, Chiang YC. Corticosteroids in the treatment of tuberculous pleurisy. A double-blind, placebo-controlled, randomized study. Chest. 1988; 94(6): 1256-1259.

20. Al-Majed SA. Study of paradoxical response to chemotherapy in tuberculous pleural effusion. Resp Med. 1996; 90(4): 211-214.

21. Tshibwabwa-Tumba E, Mwinga A, Pobee JOM, Zumla A. Radiological features of pulmonary tuberculosis in 963 HIV-infected adults at three Central African Hospitals. Clin Radiol. 1997; 52(11): 837-841.

22. Frye MD, Pozsik CJ, Sahn SA. Tuberculous pleurisy is more common in AIDS than in non-AIDS patients with tuberculosis. Chest. 1997; 112(2): 393-397

23. Sharma S, Sarin R, Khalid UK, Singla N, Sharma PP, Behera D. Clinical profile and treatment outcome of tubercular pleurisy in pediatric age group using DOTS strategy. Indian J Tuberc. 2009; 56: 191200. 